\title{
Acute Confusional State Revealing Moyamoya Disease in the Emergency Department: A Rare Entity
}

\author{
Javier Guerrero-Niño ${ }^{1}$, Sarah Uge-Ginsberg ${ }^{1}$, Pierre Marcuyez ${ }^{1}$, Pierrick Le Borgne ${ }^{1}$, Xavier Jannot $^{2}$, Noel Lorenzo-Villalba ${ }^{2}$ \\ ${ }^{1}$ Emergency Unit Department, Hôpitaux Universitaires de Strasbourg, Strasbourg, France \\ ${ }^{2}$ Service de Médecine Interne, Diabète et Maladies Métaboliques, Hôpitaux Universitaires de Strasbourg, Strasbourg, France
}

\section{Doi: 10.12890/2021_002431 - European Journal of Case Reports in Internal Medicine - ๑ EFIM 2020}

Received: 28/02/2021

Accepted: 03/03/2021

Published: $17 / 03 / 2021$

How to cite this article: Guerrero-Niño J, Uge-Ginsberg S, Marcuyez P, Le Borgne P, Jannot X, Lorenzo-Villalba N. Acute confusional state revealing moyamoya diesase in the emergency department: a rare entity. EJCRIM 2021;8: doi:10.12890/2021_002431.

Conflicts of Interests: The Authors declare that there are no competing interests.

This article is licensed under a Commons Attribution Non-Commercial 4.0 License

\section{ABSTRACT}

A 54-year-old woman was admitted to the emergency department for an acute, fluctuating altered mental status and reduced perceptual awareness of her surroundings as well as disorganized thinking. Blood tests, including for drugs, were normal. A CT scan of the brain was normal. Magnetic resonance imaging and CT angiography of the supra-aortic vessels were both were consistent with moyamoya disease. The patient was hospitalized for further investigations.

\section{LEARNING POINTS}

- Moyamoya disease should be considered in the differential diagnosis of middle-aged patients presenting with an acute confusional state of unknown aetiology in the emergency department.

- The absence of focal examination findings does not exclude neurological disease as the cause of acute confusion, requiring further neuroimaging tests.

\section{KEYWORDS}

Confusional state, magnetic resonance imaging, moyamoya disease

\section{INTRODUCTION}

Delirium and confusional states are associated with many complex underlying medical conditions and can be difficult to diagnose. These conditions include common mental disorders encountered in medical practice and particularly among elderly individuals.

\section{CASE DESCRIPTION}

A 54-year-old woman was admitted to the emergency department for an acute, fluctuating altered mental status with reduced perceptual awareness of her surroundings as well as disorganized thinking since early that morning. The patient was not agitated and did not have hallucinations. Her relatives confirmed that she was in her usual state of health the previous night.

The patient had no history of fever, chills, cough, shortness of breath or weight loss. There were no newly introduced medications. Her medical history was significant for hypertension and type 2 diabetes mellitus, treated with metformin, nebivolol and hydrochlorothiazide. Her family history was non-contributory.

She had been born in Vietnam and settled in France in 2004. She had no known drug or food allergies, as well as no history of smoking, alcohol abuse or illicit drug use. 
On physical examination, her heart rate was 84 beats/min, blood pressure was 100/80 mmHg, respiratory rate was 20 breaths/min, and oxygen saturation was $95 \%$ on room air. The patient was alert but confused as to time and space. Cranial nerve examination was normal, and no focal neurological deficits were found. Heart sounds were regular without any rubs or murmurs. The lungs were clear.

Blood tests showed leucocytosis $\left(11 \times 10^{9} / \mathrm{I}\right)$ with $7.51 \times 10^{9} / \mathrm{I}$ polymorphonuclear neutrophils and negative C-reactive protein. The glomerular filtration rate was $97 \mathrm{ml} / \mathrm{min}$ and serum creatinine was $62.5 \mu \mathrm{mol} / \mathrm{l}$. Electrolytes were within the normal ranges (sodium, potassium, chloride, calcium, magnesium and phosphate) and blood glucose was $1.1 \mathrm{~g} / \mathrm{l}$. Blood gases were normal. Coagulation, liver and thyroid tests were within normal ranges. Urinalysis was negative. A computed tomography (CT) scan of the brain was normal. The electroencephalogram showed slow electrical activity in the left hemisphere without signs of epileptic seizure.

The investigations were completed with cerebral magnetic resonance imaging (MRI) (Fig. 1) which showed multiple recent ischaemic lesions in the entire left Sylvian territory, absence of visible flow within the explored portion of the right internal carotid artery as well as in the entirety of the right middle cerebral artery, occlusion of the M1 segment, and absence of flow of the entirety of the left middle cerebral artery.

Following MRI, CT angiography of the supra-aortic vessels (Fig. 2) revealed narrowing of the right internal carotid artery in its post-bulbar portion, occlusion of the terminal portion of the right internal carotid artery, with partial recovery of the circulation of the right middle cerebral artery via the collaterals. There was narrowing with sub-occlusive diffuse stenosis of the M1 segment of the left middle cerebral artery as well as the P2/P3 portion of the right posterior cerebral artery; the anterior cerebral arteries, internal carotid artery and vertebral arteries were patent. Moyamoya disease (MMD) at the level of the M1 segments of the cerebral middle arteries was diagnosed.

The patient was hospitalized in the neurology department for further investigations.

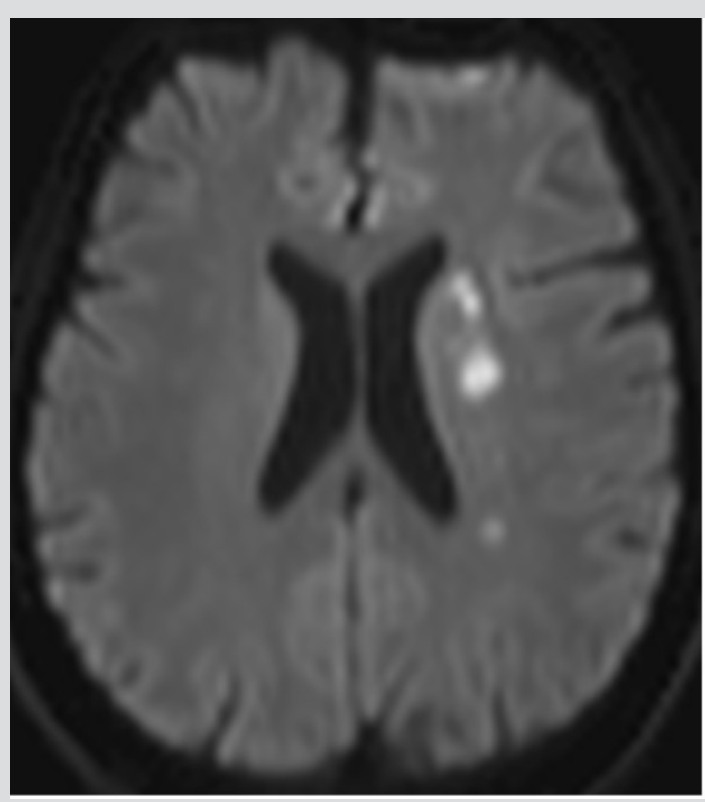

Figure 1 Cerebral magnetic resonance image

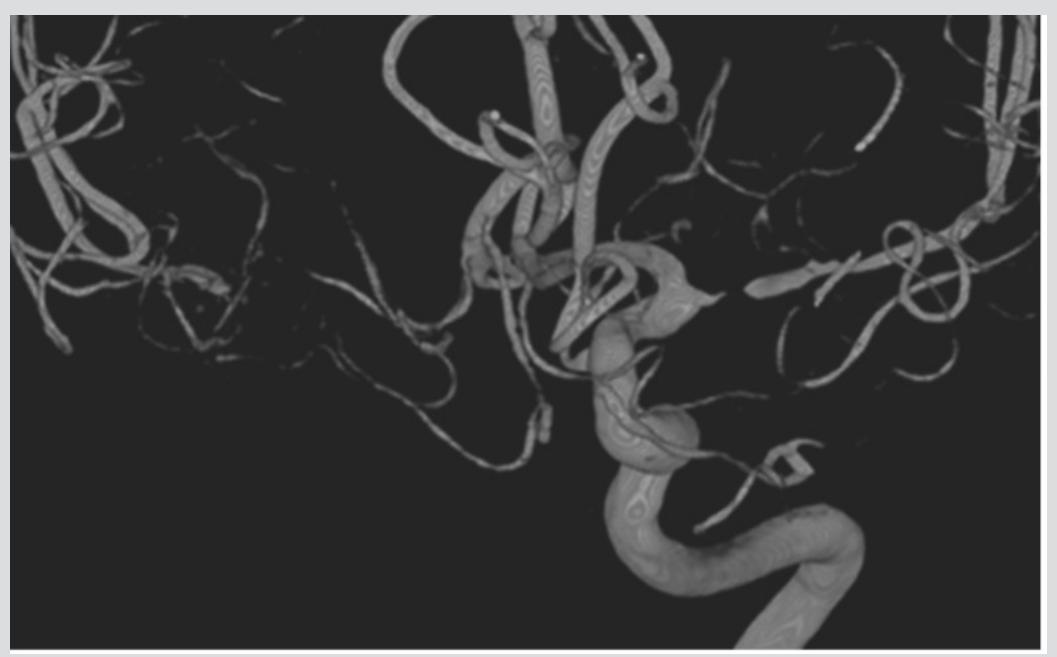

Figure 2 Angiography of the supra-aortic vessels

\section{DISCUSSION}

Acute confusional state may be caused by a wide range of medical conditions. In the case presented, fluid and electrolyte disturbances (dehydration, hyper- or hyponatraemia, hyper- or hypocalcaemia), infections (respiratory, urinary, skin and soft tissue), organ failure and hypoglycaemia were ruled out. Other causes such as drug toxicity, drug poisoning, or withdrawal from alcohol and sedatives were also ruled out $^{[1]}$.

The absence of focal neurological examination findings did not exclude the possibility of a neurological disease ${ }^{[2]}$ as the cause of the acute confusional state. As no obvious cause was identified, further testing such as a magnetic resonance imaging was performed, which helped establish the diagnosis in this case. 
The prevalence of MMD varies globally but is higher in Asian countries ${ }^{[3]}$. Its cause is still unknown. The disease is characterized by vessel wall thickening and angiogenesis. MMD can affect children and adults, showing a bimodal distribution in the age of onset. In adults, the peak has been described at age 40. When the disease appears in the setting of other associated conditions, it is referred to as moyamoya syndrome (MMS). This syndrome includes haematological, metabolic, vasculitic and autoimmune disease and genetic disorders ${ }^{[3]}$. In our case, we were not able to establish the presence of an associated condition in the emergency department.

The clinical presentation of MMD primarily includes ischaemic stroke and transient ischaemic attack; intracerebral, intraventricular and subarachnoid haemorrhage; and seizures [3,4]. Other clinical manifestations include headache, dystonia, chorea or dyskinesia, although the latter are uncommon. In addition, the disease has been incidentally found in patients undergoing neuroimaging studies for other medical reasons.

CT scanning of the brain is helpful in the diagnosis of cerebral infarction involving cortical and subcortical regions, although these patterns are not specific for this disease. In addition, the following MRI findings allow identification of vascular changes in moyamoya: (a) dilated collateral vessels in the basal ganglia or thalamus; (b) focal, tubular or serpentine hyperintensities on FLAIR or contrast-enhanced T1 images in the subarachnoid spaces; and (c) prominent hypointensity in medullary veins draining areas of impaired cerebral perfusion on susceptibility weighted imaging $(\mathrm{SWI})^{[5,6]}$.

The final diagnosis is based on the characteristic angiographic appearance of bilateral stenosis affecting the distal internal carotid arteries and proximal circle of Willis vessels, along with the presence of collateral vessels. However, definitive diagnosis requires: (a) stenosis or occlusion at the terminal portion of the internal carotid artery and at the proximal portion of the anterior and middle cerebral arteries; (b) abnormal vascular networks in the basal ganglia which can also be diagnosed by the presence of multiple flow voids on brain MRI; and (c) angiographic findings present bilaterally, while cases with unilateral angiographic findings are considered probable ${ }^{[7]}$.

\section{CONCLUSIONS}

Confusional states present a diagnostic dilemma in the emergency department. MMD is a rare entity but could be considered in the differential diagnosis of middle-aged patients presenting with an acute confusional state in the emergency department.

\section{REFERENCES}

1. Roche V. Southwestern Internal Medicine Conference. Etiology and management of delirium. Am J Med Sci 2003;325:20.

2. Oldenbeuving AW, de Kort PL, Jansen BP, Algra A, Kappelle LJ, Roks G. Delirium in the acute phase after stroke: incidence, risk factors, and outcome. Neurology 2011;76(11):993-999.

3. Kim JS. Moyamoya disease: epidemiology, clinical features, and diagnosis. J Stroke 2016;18:2.

4. Roach ES, Golomb MR, Adams R, Biller J, Daniels S, Deveber G, et al; American Heart Association Stroke Council; Council on Cardiovascular Disease in the Young. Management of stroke in infants and children: a scientific statement from a Special Writing Group of the American Heart Association Stroke Council and the Council on Cardiovascular Disease in the Young. Stroke 2008;39(9):2644-2691.

5. Azizyan A, Sanossian N, Mogensen MA, Liebeskind DS. Fluid-attenuated inversion recovery vascular hyperintensities: an important imaging marker for cerebrovascular disease. AJNR Am J Neuroradiol 2011;32:1771.

6. Yu X, Yuan L, Jackson A, Sun J, Huang P, Xu X, et al. Prominence of medullary veins on susceptibility-weighted images provides prognostic information in patients with subacute stroke. AJNR Am J Neuroradiol 2016;37(3):423-429.

7. Research Committee on the Pathology and Treatment of Spontaneous Occlusion of the Circle of Willis, Health Labour Sciences Research Grant for Research on Measures for Infractable Diseases. Guidelines for diagnosis and treatment of moyamoya disease (spontaneous occlusion of the circle of Willis). Neurol Med Chir (Tokyo) 2012;52:245. 